\title{
La ri-scrittura del patrimonio culturale nell'Era digitale
}

\author{
Rosa Maria Giusto y Mario Buono \\ National Research Council Italy (CNR)-Institute for Research on Innovation and Services for Development (IRISS), Napoli \\ Università della Campania «Luigi Vanvitelli» \\ r.giusto@iriss.cnr.it; mario.buono@unicampania.it
}

«Punto di riferimento dinamico» di una comunità in costante mutamento, il patrimonio culturale include «paesaggi, luoghi storici, siti e ambienti costruiti dall'uomo, così come la biodiversità, le collezioni, le pratiche culturali del passato e del presente, le esperienze di vita e la conoscenza» (ICOMOS, 1999; Battilani, 2019) chiamando in causa processi di riconoscimento e di condivisione legati alla necessità di rispondere ai bisogni identitari del presente. II superamento di una «concezione puramente architettonica del patrimonio culturale a favore di una visione antropologica, multifunzionale e universale», sancito dall'Unesco nel 1994, ha avuto il merito di ampliare considerevolmente il significato e gli ambiti di pertinenza del patrimonio culturale. In quanto bene universale, esso è soggetto alle attenzioni e alla cura che tutti, nessuno escluso, possono apportarvi, coinvolgendo il visitatore nelle fasi di riconoscimento e salvaguardia attiva del bene; allo stesso tempo, anche in presenza di beni o categorie riconducibili a specifici usi e pratiche culturali-come nel caso emblematico del patrimonio religioso- essi rientrano comunque in un'idea di eredità comune -da cui discende la definizione di heritage-, «transnazionale» e «trans-spirituale», identitaria dell'intera umanità. Tale approccio ha determinato come immediata conseguenza un processo di democratizzazione delle conoscenze non più destinate esclusivamente a comunità di studiosi e specialisti della materia ma invece rivolte a tutti coloro che ne fossero a vario titolo interessati, aprendo al delinearsi del turismo culturale quale risorsa economica e territoriale globale [1] y [2]. II solido legame che intercorre tra cultura e turismo nella società contemporanea è ben rappresentato dal ruolo che la prima esercita nel campo dell'innovazione turistica legata alla progettazione di percorsi partecipati ed esperienziali di fruizione del patrimonio culturale e dall'importanza che, a sua volta, il turismo riveste nelle politiche di valorizzazione, finanziamento e divulgazione delle conoscenze (Battilani, 2019). II necessario processo d'identificazione e riconoscimento da parte dei visitatori dei contenuti culturali di cui il patrimonio culturale è portavoce induce a interrogarsi sulle modalità più efficaci di trasmissione e fruizione di tali beni, soprattutto in considerazione dell'ingresso del digitale e dell'loT -Internet of Things- (Ashton, 2009) nel

1. Lecce Experience, prototipo sperimentale di ricerca realizzato nell'ambito dello Stream INMOTO-INformation and MObility for TOurism

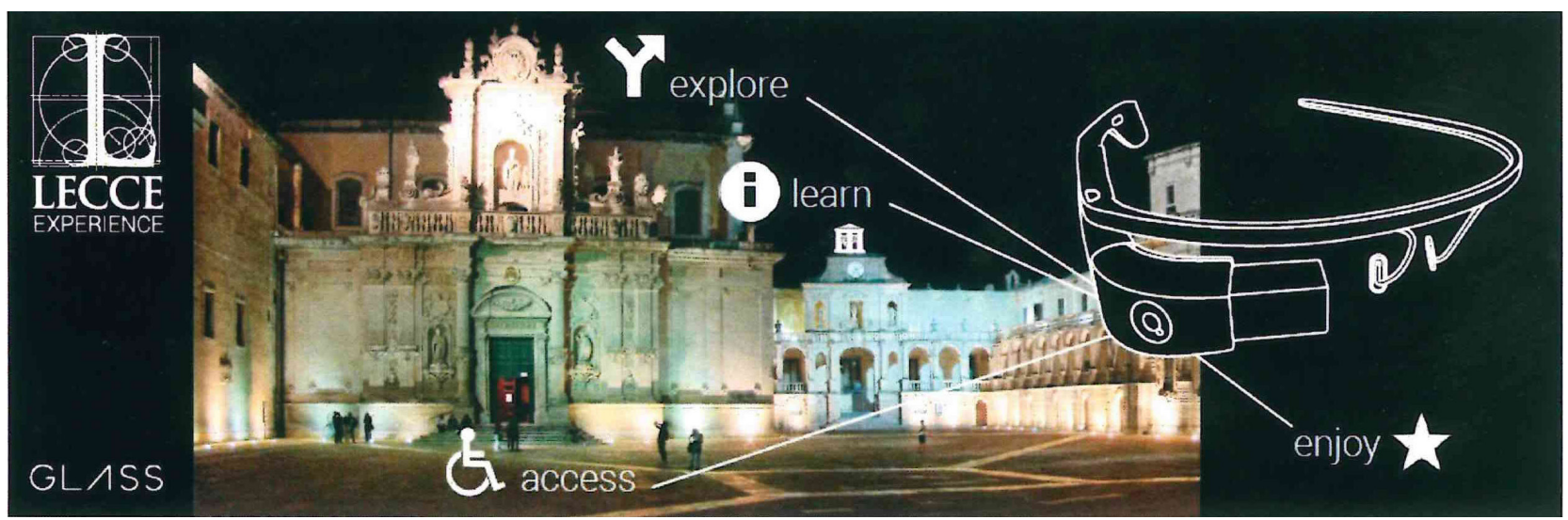




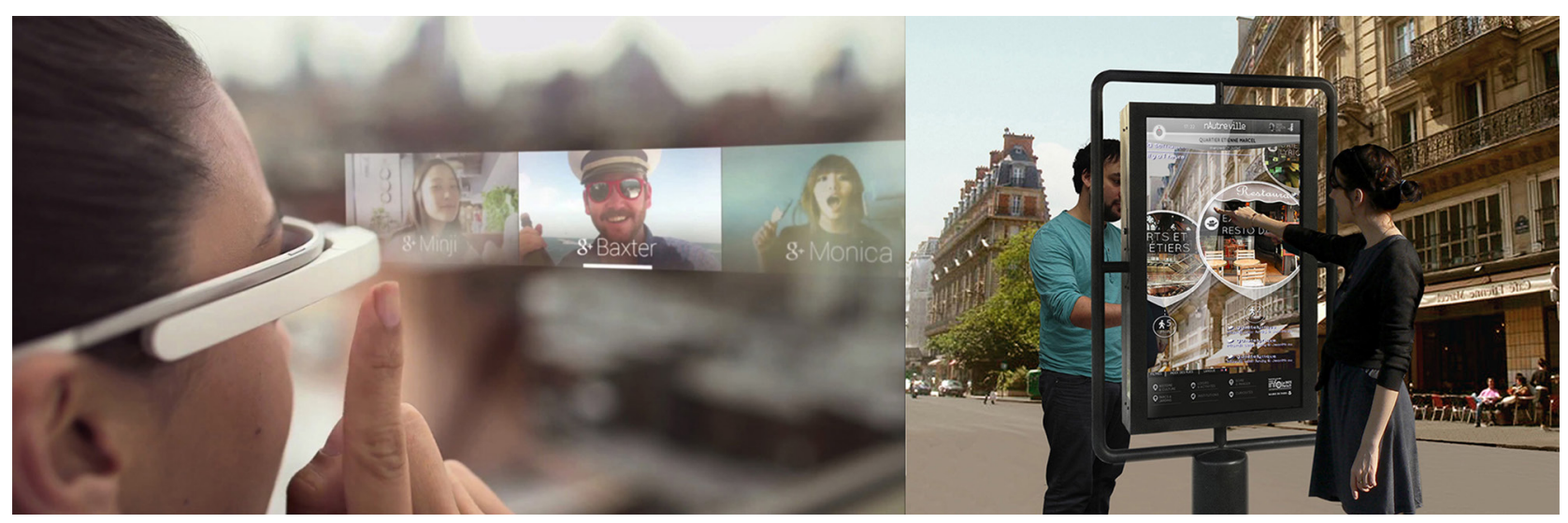

2. Tecnologia google glass per la fruizione assistita e pensilina high-tech «nAutreVille», con pannello per la realtà aumentata interattivo e girevole, progettata da Maria Laura Mendez

settore dei beni culturali e dell'ampliamento di nuovi pubblici. Nel processo di narrazione del patrimonio culturale, nuovi mezzi e modalità di trasmissione sono infatti intervenuti incidendo sensibilmente sul sistema di trasferimento di conoscenze e di contenuti culturali veicolati dal patrimonio culturale. Uno degli aspetti più interessanti derivante da tali nuovi assetti consiste nell'analizzare e prefigurare il modo in cui sarà possibile combinare digitale e loT con i sistemi di narrazione «consolidati», aprendo a nuovi sviluppi e nuove applicazioni. Un ruolo fondamentale in tal senso è rappresentato dall'innovazione che costituisce il driver principale di sviluppo e di collegamento tra gli elementi più legati alla tradizione e quelli derivanti dall'uso delle nuove tecnologie [3].

Nell'ambito delle nuove modalità di narrazione del patrimonio culturale, un ruolo particolare riveste il "genere» delle guide turistiche che occupa uno specifico campo di applicazione riferito al processo d'interpretazione e traduzione dei valori di un sito o di un bene culturale tout court. "Molto più che per il turismo stanziale [...] o del divertimento [...], le guide diventano uno strumento imprescindibile perché svolgono quel ruolo di «mediazione culturale» [... $]$ (Cohen, 1985) spiegazione e compendio che facilita l'esperienza dei turisti» (Tramontana, 2010: 44). Un tempo unicamente prodotte in formato cartaceo tascabile, sono state progressivamente affiancate e in parte sostituite da supporti vocali che di fatto riproducono le "caratteristiche sintattiche e stilistiche» (Palombini, 2012: 11) in precedenza affidate al testo scritto, fino ad arrivare alla progettazione di sistemi di narrazione digitale del patrimonio culturale sempre più complessi e articolati al punto di aver originato un nuovo, specifico, campo di studio e di applicazione denominato digital storytelling (2012: 10). Non si tratta di «semplici» ricostruzioni digitali di monumenti, siti o reperti -di cui se ne prevede la sostituzione in assenza degli originali- ma di un complesso di strategie e tecniche necessarie a rendere la comunicazione e la trasmissione del patrimonio culturale quanto più inclusive e coinvolgenti, secondo un approccio narrativo che sposta il baricentro del progetto di comunicazione dall'oggetto -il bene, il monumento, il sito narrato, ma anche i riti e le testimonianze immateriali- al soggetto visitatore che non è più il consumatore passivo di messaggi e prodotti culturali pre-determinati e pre-confezionati, ma un protagonista pienamente coinvolto nel processo di comprensione e ri-significazione del bene narrato. Alla base di tale approccio vi è la necessità di coniugare la comunicazione del valore di un luogo o di un particolare bene con l'esigenza di renderlo «appetibile» e trasmissibile, rendendo comprensibili aspetti altrimenti non pienamente trasferibili, suscitando interesse e curiosità nei visitatori, stimolati e supportati nel goderne pienamente (Tramontana, 2010: 44).

Proprio in riferimento al tema dell'heritage tourism, l'ICOMOS ha introdotto, a partire dal 2002 (ICOMOS, 2008; Silberman, 2006), «sette principi per una valida attività di interpretazione» (Tramontana, 2010: 44) dei siti culturali che comprendono significativamente: Accesso e comprensione; Fonti informative; Contesto e ambiente; Autenticità; So- 


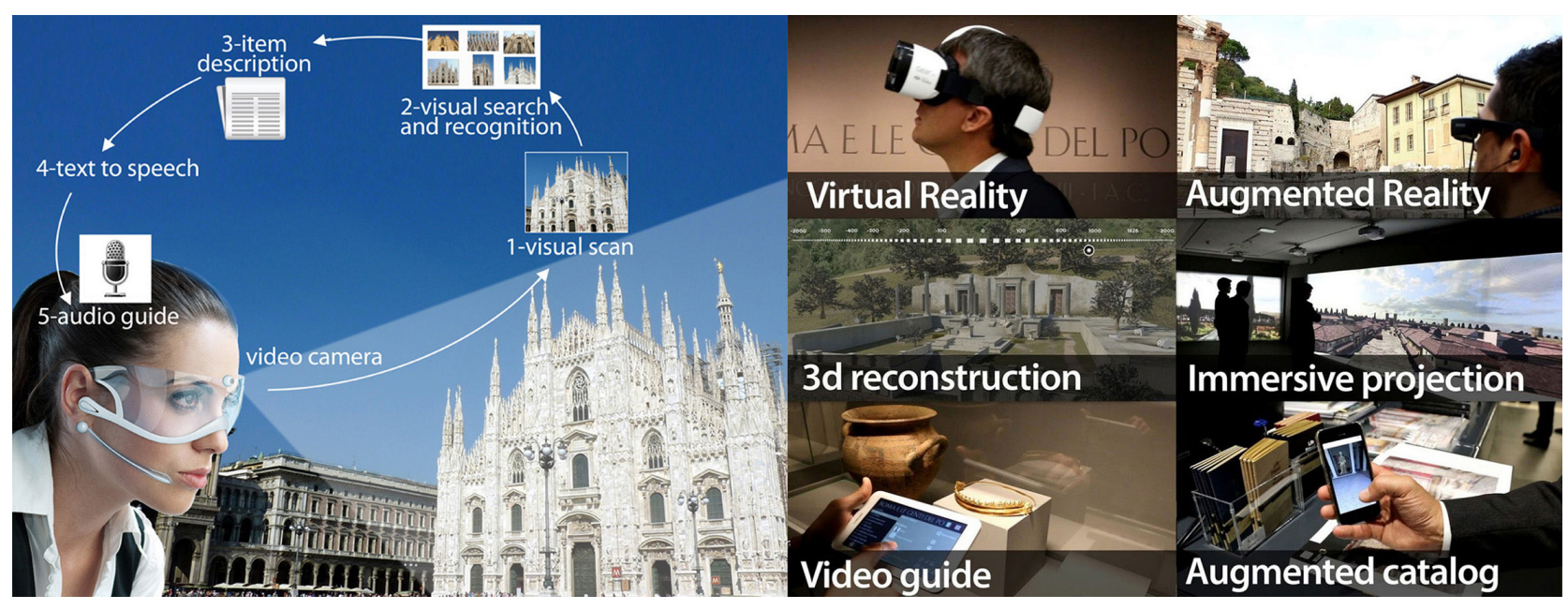

3. Audio Guide Mobile e Tecnologie di realtà virtuale e aumentata, integrate nel progetto Brixia Time Machine, vincitore del premio AVICOM 2016 a Budapest

stenibilità; Inclusività; Ricerca, formazione e valutazione. Tali principi si ritrovano aggiornati e rielaborati negli obiettivi del Programma «Horizon 2020» (2014-2020) per la ricerca e l'innovazione che li ha significativamente «tradotti» e sintetizzati in tre «parole-chiave» indispensabili per una crescita: Intelligente, Sostenibile e Inclusiva (Horizon 2020). L'incidenza dell'approccio narrativo quale medium in grado di veicolare la trasmissione di contenuti culturali e la loro corretta ricezione da parte degli utenti, comporta alcune considerazioni riguardanti: -la necessità di coinvolgere emotivamente l'utente, rendendolo parte di una narrazione partecipata e condivisa con la comunità e pertanto radicata nel riconoscimento di propri valori identitari; -il timore d'ingenerare confusione tra codice e contenuto, con la conseguente necessità di fornire un'informazione scientifica, non semplicemente «verosimile», ma storicamente attendibile e plausibile quale vero plusvalore del progetto di comunicazione proposto; -la consapevolezza di dover includere il potenziale didattico-educativo tra le finalità dell'approccio narrativo dove edu-tainment e entertainment convivono in una precisa strategia comunicativa che supera il dato meramente "aneddotico" per favorire la costruzione di una coscienza condivisa del bene che implichi il coinvolgimento del pubblico a molteplici livelli (tematico, filologico, esperienziale) incidendo significativamente sul riconoscimento e sull'attrattività del bene stesso.

Le innovazioni introdotte dalle nuove tecnologie digitali nell'ambito del digital storytelling riguardano principal- mente l'immersività e l'interazione, fattori che consentono all'utente di «influenzare con il proprio comportamento il flusso di una comunicazione» (Palombini, 2012: 17) [4]. Modificando strutture e tecniche narrative consolidate e facendone sorgere di nuove, quali «la multilinearità e la ciclicità dell'esperienza» (17), tali sistemi rimettono in discussione la stessa struttura organizzativa e «sintattica» delle guide turistiche e dei circuiti museali, costringendoci a fare i conti con la necessità di dover entrare in «un'ottica creativa molto diversa da ciò che siamo abituati a pensare come "narrazione"» (17). Se nell'impostazione tradizionale di visita «l'utente viveva una vicenda composta da una sequenza precisa e immutabile di eventi, ora può scegliere diversi percorsi [...] alternativi, le cui possibili combinazioni andranno tutte previste e realizzate con attenzione» (17), offrendo enormi sviluppi anche sul piano dell'apprendimento. Poiché le nuove tecnologie "consentono di disseminare informazioni [...] non [...] più legate a un singolo reperto decontestualizzato» (17) ma alla costruzione di percorsi di visita e conoscenza personalizzati, si apre la possibilità concreta di prevedere approfondimenti tematici funzionali al grado di formazione, di conoscenza, d'istruzione e alla stessa professione di ciascun visitatore (e dunque agli interessi degli utenti, tanto da poter parlare di "costumizzazione» anche per il settore delle guide e delle esperienze di visita), mediante la creazione di «biblioteche» personalizzate e playlist specifiche degli argomenti privilegiati dagli utenti, da rive- 


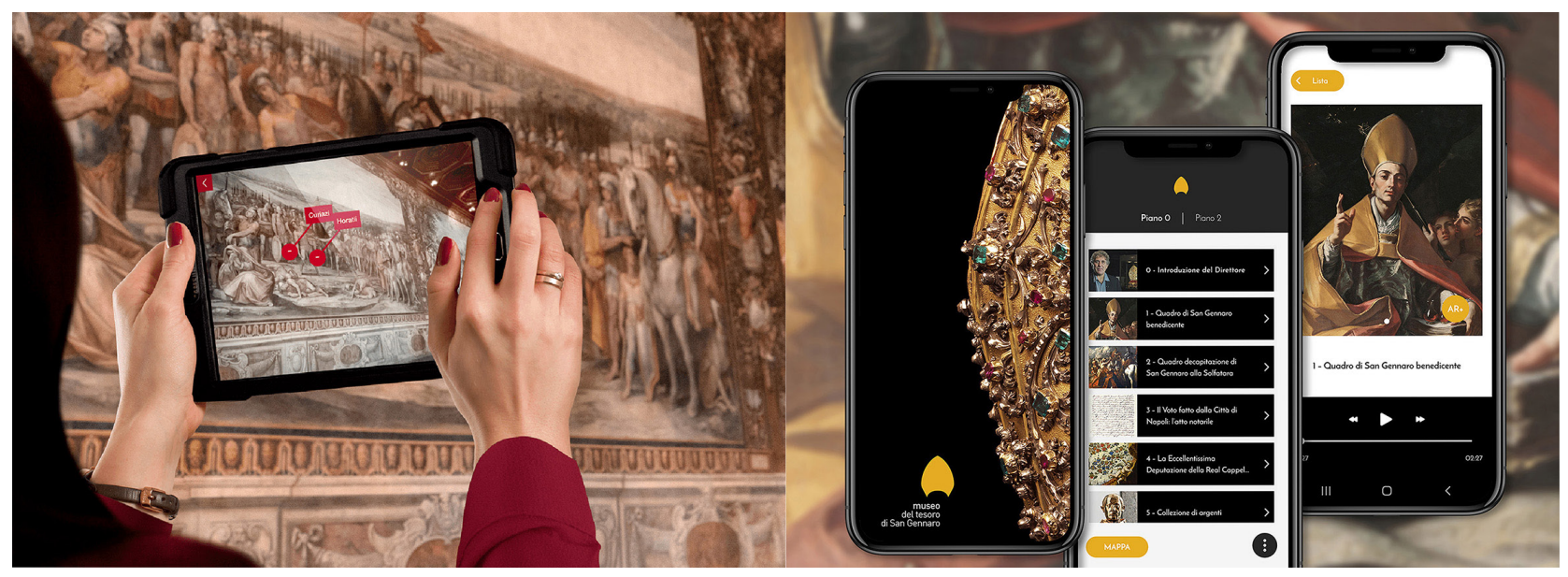

4. Video guida interattiva di Musei in Comune e i Segreti del Tesoro di San Gennaro in una guida interattiva - ETT Solutions

dere e ripercorrere anche a conclusione del percorso, con l'impareggiabile opportunità di proseguire l'esperienza di apprendimento anche al di fuori del circuito di visita. Senza contare che la guida ha anche una funzione "predittiva»: consultata prima che il viaggio o l'itinerario si compia anticipa la fruizione del sito o dell'opera narrata, consentendo di pre-figurarne l'esperienza. «In nessun'altra tipologia testuale [...] la valorizzazione dello spazio[/bene descritto] si correlaziona in maniera tanto forte con la costituzione dell'identità del soggetto lettore [...] che compirà il suo percorso leggendolo e attraversandolo» (Ragonese, 2010: 7). Accanto a una dimensione spaziale, infatti, il patrimonio ha anche una dimensione temporale «che lo proietta nel mondo della storia e della sua interpretazione» (Battilani, 2019). Attraverso «una serie di elementi sintetizzabili nel concetto di «simulazione»» (Palombini, 2012: 20), sarà possibile «in modo sempre più dettagliato e realistico, [...] riprodurre micro e macro-processi storici tenendo conto delle variabili sociali che li condizionarono» (20) determinando forme di «narrazione procedurale [...] generate [...] dagli utenti stessi, sul canovaccio di fattori sociali significativi e verosimili. Ciò sarà [...] di grande potenzialità per la comprensione di dinamiche e processi di trasformazione sociale» (20). Lo sviluppo delle ricostruzioni virtuali, inoltre, potrà comportare la possibilità di condividere modelli digitali più o meno dettagliati del repertorio espositivo di ciascun museo consentendo la creazione di «vasti database diffusi» (21) utili per la ricostruzione sempre più approfondita e aggiornata di monumenti, siti e processi trasformativi inerenti al patrimonio culturale.

Un ulteriore sviluppo del digital storytelling potrà aversi proprio nel campo della qualità delle informazioni trasmesse attraverso i muovi strumenti. In altre parole, sarebbe auspicabile il passaggio dal digital storytelling al digital history-telling, dove i nuovi strumenti digitali potranno essere impiegati per rendere più "godibile» la narrazione delle informazioni storiche inerenti ciascun itinerario di visita, sia esso relativo a un sito o al percorso espositivo d'un museo. Detto altrimenti, ciò che dovrà modificarsi saranno i modi attraverso i quali comunicare e trasmettere alla collettività la conoscenza e la storia del patrimonio culturale, del quale bisognerà prevedere diversi livelli di approfondimento, funzione della diversità del pubblico al quale ci si rivolge. Mutando il pubblico muta, infatti, la qualità delle domande poste e, di conseguenza, il grado di approfondimento delle risposte attese, necessitando di un sistema di informazioni quanto più flessibile, inclusivo, aperto e dinamico che consenta di variare la «scala» e l'ampiezza delle conoscenze fornite, senza per questo formulare risposte incompiute o anche solo parzialmente incomplete. La sfida futura, in tema di comunicazione delle conoscenze legate al patrimonio culturale, consisterà infatti nel reinventarsi non «il genere delle guide» ma i suoi modi di comunicazione, oggi illimitati grazie alle infinite possibilità messe a disposizione dai nuovi media e dalle tecnologie che consentono di dirimere questioni un tempo apparentemente irrisolvibili -dalle difficoltà di datazione di edifici od opere d'ar- 
te, all'analisi dettagliata delle tecniche di esecuzione, ai materiali, alle condizioni di degrado- ampliando enormemente il campo d'indagine e di sperimentazione e di conseguenza la conoscenza e la divulgazione del patrimonio culturale.

\section{Conclusioni}

«Le forme di interpretazione [e comunicazione del patrimonio culturale] non sono informazioni «a margine»» (Tramontana,
2010: 45) del percorso di visita di un sito o di un bene culturale ma rientrano in un discorso più ampio frutto di «un attento lavoro di selezione e gestione di tutte le fasi dell'esperienza» (45). II valore di una «mediazione» narrativa consapevole, integrata e potenziata dall'uso di strumenti digitali, diviene sempre più elemento centrale nelle politiche di valorizzazione e riconoscimento del patrimonio culturale dal momento che esso «non parla da solo» (45) ma ha bisogno di professionalità e strumenti informativi sempre più flessibili e aggiornati che lo disvelino e ne raccontino il significato più profondo.

\section{Bibliografia}

ASHTON, Kevin (2009), «That "Internet of Things" Thing», RFID Journal, 22 June 2009. En: <http://www.rfidjournal.com/articles/pdf?4986> (ultima consultazione: 27-02-2020).

BATTILANI, Patrizia (2019), «Si fa presto a dire patrimonio culturale. Problemi e prospettive di un secolo di patrimonializzazione della cultura», Storia e Futuro, n. ${ }^{\circ}$ 51, dicembre. En <http://storiaefuturo.eu/si-presto-dire-patrimonio-culturale-problemi-prospettive-un-secolo-patrimonializzazione-della-cultura/> (ultima consultazione: 25-02-2020).

COHEN, Erik (1985), «The Tourist Guide: The Origins, Structure and Dynamics of a Role», Annals of Tourism Research, n. ${ }^{\circ} 12$.

GIANNITRAPANI, Alice (2010), «Verso una guida transmediale. Turisti per caso», E/C, serie speciale, Anno IV, n. ${ }^{\circ}$ 6, pp. 85-98. En: <http:// www.ec-aiss.it/> (ultima consultazione: 25-02-2020).

HORIZON 2020, (2014-2020), Programma Quadro dell'Unione Europea. En: <https://www.researchitaly.it/horizon-2020/> (ultima consultazione: 22-02-2020).

ICOMOS, INTERNATIONAL CULTURAL TOURISM CHARTER (1999), Managing Tourism at Places of Heritage Significance, 12a Assemblea Generale del Messico, Ottobre 1999. En: <https://www.icomos.org/charters/tourism_e.pdf> (ultima consultazione: 25-02-2020).

ICOMOS (2008), The ICOMOS Charter for the Interpretation and Presentation of Cultural Heritage Sites, 4 October 2008. En: <http://icip. icomos.org/downloads/ICOMOS_Interpretation_Charter_ENG_04_10_08.pdf> (ultima consultazione: 27-02-2020).

PALOMBINI, Augusto (2012), «Narrazione e virtualità: possibili prospettive per la comunicazione museale», Digitalia, anno VII, n. ${ }^{\circ}$, pp. 9-22.

RAGONESE, Ruggero (2010), «Guide turistiche: un'introduzione», E/C, serie speciale, Anno IV, n. 6, pp. 5-18. En: <http://www.ec-aiss.it/> (ultima consultazione: 25-02-2020).

SILBERMAN, Neil (2006), «The ICOMOS-Ename Charter Initiative: Rethinking the Role of Heritage Interpretation in the 21st Century», The George Wright Forum, 1. En: <http://www.georgewright.org/231 silberman.pdf> (ultima consultazione: 25-02-2020).

TRAMONTANA, Andrea (2010), «ll ruolo della guida turistica nel processo di interpretazione di un sito culturale», E/C, serie speciale, Anno IV, n. ${ }^{\circ}$ 6, pp. 43-50. En: <http://www.ec-aiss.it/> (ultima consultazione: 25-02-2020).

UNESCO World Heritage Centre (1996), Report of the expert meeting on evaluation of general principles and criteria for nominations of natural World Heritage Sites, Parc national de la Vanoise, France, March 22-24. 